UC-15

Issued: July 1982

$L A--9238-M S$

DE82 021842

\title{
Robust Estimation of Standard Deviation Using Ordered Samples
}

\author{
R. Beedgen* \\ This roporl was prepared as an accounl of work sponsored by an agency of the United States Government, \\ Neither the United States Gowernment nar any agency thereot, nor any of their emplovees, makes any \\ warranty, express or impolied, or assumes eny legal lizbility ot responsibility for the accuracy, \\ completeness. or ussitalnoss of any iniormation, apparatus. product, of process disclosed, of \\ reveresents that its usk voould nol intringe privately owmed rights. Retetence hetein to any specilic

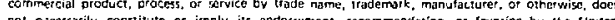 \\ not nieassorily constivun or imply its encorsintent. recommendation, or lavering by the United \\ necessarily stite or reflect thase of the United Sites Goveenment or any agency thereet.
}

-Guest Scientist. Nuclear Research Center, Karlsruhe, P. O. Box 3640, D-7500 Karlsruhe, FEDERAL REPUBLIC OF GERMANY. 


\section{ROBUST ESTIMATION OF STANDARD DEVIATION \\ USING ORDERED SAMPLES}

by

R. Beedgen

\section{ABSTRACT}

A robust estimate for the standard deviation of a normal distribution is developed. We choose the contaminated normal distribution as our outlier model and demonstrate the method with two examples representing data from international interlaboratory experiments.

\section{INTRODUCTION}

In this report, we present an estimation function for the standard deviation of a normal distribution. This estimation function is compared with two classical methods under the assumption of a pure normal distribution as well as a contaminated normal distribution. We show that the estimation method is useful when there are outliers among the data that are the result of bad measurements.

The estimation methods are compared with the help of the asymptotic relative efficiency. Using the contamination model, we demonstrate the robustness of our method. Two examples are used to explain the estimation method. The data came from international interlaboratory comparisons. These programs investigated the measurement precision for ${ }^{238} \mathrm{~J}$. During the evaluation of the data, a heuristic application of the estimation method was successfully used. This application was based on the graphical interpretation of the estimation method. 


\section{PRELIMINARY REMARKS}

Let $\mathrm{N}$ independent and identically distributed (i.i.d.) random variables (rv's) on a probability space $(\Omega, A, P)$ with a normal distribution be given:

$$
x_{i} \sim N(\mu, \sigma), \quad i=1,2, \ldots, N
$$

Let us denote the distribution function (df) of each of these $r v^{\prime} s$ by $F(x)$. Now we define the empirical distribution function as

$$
\begin{aligned}
F_{N}^{*}(t): & \left.=\text { (number of } x_{i} \leq t, 1 \leq i \leq N\right) / N \\
& =\sum_{i=1}^{N} I_{X_{i} \leq t} / N,
\end{aligned}
$$

where I stands for the indicator variable. Now, if we $f i x t \in R$,* $F_{N}(t)$ is a $r v$ that stands for the arithmetic mean of the independent indicator variables $I_{X_{i} \leq t}$. We have

$$
E\left(I_{X_{i} \leq t}\right)=P\left\{x_{i} \leq t\right\}=F(t)
$$

According to the weak law of large numbers, we have

$$
\lim _{N \rightarrow \infty} P\left\{\sup _{t \in R_{N}}\left|F_{N}^{*}(t)-F(t)\right| \leq \varepsilon\right\}=1 \text {, }
$$

which means that it is reasonable to use the empirical distribution function for estimation of sample quantiles.

${ }^{\star} \mathrm{R}:=$ set of real numbers. 
III. ESTIMATION METHOD FOR THE STANDARD DEVIATION $\sigma$

First, we define with the help of the rv's $x_{1}, x_{2}, \ldots$ $\mathrm{x}_{\mathrm{N}}$ new rv's. We assume $\mathrm{N}=2 \mathrm{n}$ and define

$$
\begin{aligned}
z_{k}=\left|x_{i_{k}}-x_{j_{k}}\right|, & k=1,2, \ldots, n ; \\
& i_{k}, j_{k} \in\{1,2, \ldots, N\} ; \\
& i_{k} \neq j_{k} ; \text { for } k \neq k \text { we have } \\
& \left\{i_{k}, j_{k}\right\} \cap\left\{i_{k}, j_{k},=g\right.
\end{aligned}
$$

Now $z_{1}, z_{2}, \ldots, z_{n}$ are i.i.d. rv's.

For the df of $z_{1}$, we have

$$
H(z)=\left\{\begin{array}{cc}
2 \Phi[z /(\sqrt{2} \sigma)]-1 & \text { for } z \geq 0 \\
0 & \text { otherwise }
\end{array},\right.
$$

where $\Phi(*)$ denotes the df of the standard normal distribution. From Eq. (6), we have

$$
H(\sigma)=2 \Phi(1 / \sqrt{2})-1=: q_{\sigma},
$$

where

$$
q_{\sigma}=0.52 .
$$


If $h(z)$ is the density of $H(z)$, then

$$
h(z)=\left\{\begin{array}{cc}
\exp \left[-z^{2} /\left(4 \sigma^{2}\right)\right] /(\sqrt{\pi \sigma}) & \text { for } z>0 \\
0 & \text { otherwise }
\end{array}\right.
$$

As a result, we are able to say that $H(z)$ is a strictly increasing df in $R^{+}$and $\sigma$ is the $q_{\sigma}$-quantile of $H(z)$.

If we have the simple random sample $z_{1}, z_{2}, \ldots, z_{n}$, then the sample function $z(k), 1 \leq k \leq n$, denotes the $k^{t h}$ position of the ordered sample; that is,

$$
z_{(1)} \leq z_{(2)} \leq z_{(3)} \leq \cdots \leq z_{(k)} \leq \cdots \leq z_{(n)}
$$

If we look at Fig. 1 and Eq. (7), a suggestion for the estimation of $\sigma$ is $H^{-1}\left(q_{\sigma}\right)$, which is single-valued because $H(z)$ is strictly monotonic. But because we do not know $\sigma$, we are not able to evaluate $\mathrm{H}(\mathrm{z})$. So we help ourselves with the empirical distribution function, that is, with the inverse function of $\mathrm{H}_{\mathrm{n}^{\prime}}^{*}$ which is defined as

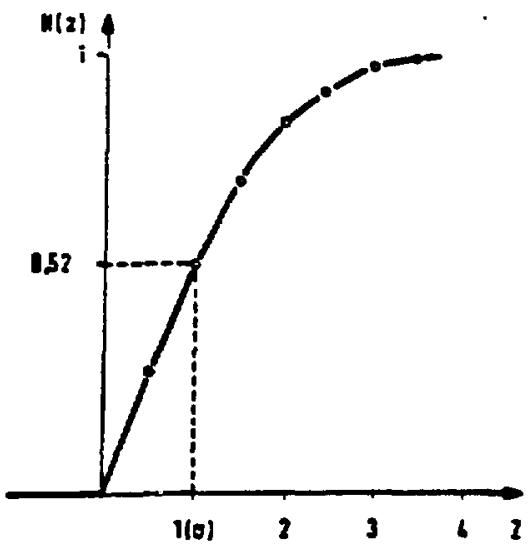

Fig. 1 .

Example of the df $\mathrm{H}(\mathrm{z})$ for $\sigma=1$. 


$$
Q_{n}^{*}(u):=H_{n}^{*-1}(u)=\inf \left\{z \mid H_{n}^{*}(z) \geq u\right\} \text { for } 0 \leq u \leq 1 \text {. }
$$

In our case, this line of reasoning leads to the following definition of an estimation function for $\sigma$ :

$$
\hat{\sigma}_{N}:=Q_{N / 2}^{*}\left(q_{\sigma}\right)
$$

Now $\hat{\sigma}_{N_{*}}$ should be analyzed. In this connection, it is very helpfu, that $Q_{n}^{*}(u)$ can be written as order statistics. We have

$$
Q_{n}^{*}(u)=z_{(j)}
$$

where $(j-1) / n<u \leq j / n$ and $j \in\{1,2, \ldots, n\} .1$ For $(j-1) / n$ $<u<j / n$, we can write $Q_{n}^{*}(u)=z_{([n u]+1)}$. The [nu] denotes the largest integer that is smaller than or equal to nu. With the help of Eq. (12), we can write $\hat{\sigma}_{N}$ as

$$
\hat{\sigma}_{\mathrm{N}}=\mathrm{z}\left(\left[q_{\sigma} \cdot \mathrm{N} / 2\right]+1\right) .
$$

The estimation function $\hat{\sigma}_{N}$ is asymptotically normally

$$
N\left[\sigma, \frac{\sqrt{q_{\sigma}\left(1-q_{\sigma}\right) / n}}{h(\sigma)}\right]
$$

distributed. Furthermore, we have

$$
\lim _{N \rightarrow \infty} P\left\{\left|\hat{\sigma}_{N}-\sigma\right|>\varepsilon\right\}=0 \text {, for all } \varepsilon>0 \text {; }
$$


that is, $\hat{\sigma}_{N}$ is a consistent estimator for $\sigma$. Furthermore, we can show that $\hat{\sigma}_{N}$ ¿s an asymptotically unbiased estimator for $\sigma .{ }^{2}$ Together with Eq. (15), we get the asymptotic variance of $\hat{\sigma}_{\mathrm{N}}$ :

$$
\begin{aligned}
& \lim _{N+\infty} N \cdot \operatorname{Var}\left(\hat{\sigma}_{N}\right)=2 \pi \sigma^{2}\left|\frac{[2 \Phi(1 / \sqrt{2})-1][2-\Phi(1 / \sqrt{2})]}{\exp (-1 / 2)}\right| \\
& =2.590^{2} \text {. }
\end{aligned}
$$

IV. CONFIDENCE INTERVAL FOR $\sigma$ WITH $\hat{\sigma}_{N}$

The problem of finding a confidence interval for $\sigma$ to the level $1-\alpha$ can be sketched in the following way: ${ }^{3}$ find numbers $k<m$ such that

$$
\mathrm{p}\left\{\mathrm{z}_{(\mathrm{k})} \leq \sigma \leq \mathrm{z}_{(\mathrm{m})}\right\}=1-\alpha
$$

is valid. The event $\{z(k) \leq \sigma\}$ can be described as the union of the events $\left\{z_{(k)} \leq \sigma \leq z_{(m)}\right\}$ and $\left\{\sigma>z_{(m)}\right\}$; that is,

$$
\begin{aligned}
& \mathrm{P}\left\{\mathrm{z}_{(\mathrm{k})} \leq \sigma\right\}=\mathrm{P}\left\{\mathrm{z}_{(\mathrm{k})} \leq \sigma \leq \mathrm{z}_{(\mathrm{m})}\right\}+\mathrm{P}\left\{\mathrm{z}_{(\mathrm{m})}<\sigma\right\} \text { or } \\
& \mathrm{P}\left\{\mathrm{z}_{(\mathrm{k})} \leq \sigma \leq \mathrm{z}_{(\mathrm{m})}\right\}=\mathrm{P}\left\{\mathrm{z}_{(\mathrm{k})} \leq \sigma\right\}-\mathrm{P}\left\{\mathrm{z}_{(\mathrm{m})}<\sigma\right\} .
\end{aligned}
$$

Because $H(z)$ is strictly increasing, we have

$$
\begin{aligned}
& \left\{z_{(k)} \leq \sigma\right\}=\left\{H\left(z_{(k)}\right) \leq H(\sigma)\right\}=\left\{H\left(z_{(k)}\right) \leq q_{\sigma}\right\} \\
& \left\{z_{(m)}<\sigma\right\}=\left\{H\left(z_{(m)}\right)<H(\sigma)\right\}=\left\{H\left(z_{(m)}\right)<q_{\sigma}\right\},
\end{aligned}
$$


and with that

$$
P\left\{z_{(k)} \leq \sigma \leq z_{(m)}\right\}=P\left\{H\left(z_{(k)}\right) \leq q_{\sigma}\right\}-P\left\{H\left(z_{(m)}\right)<q_{\sigma}\right.
$$

Now, we know that $[H(Z(1)), H(Z(2)), \ldots, H(z(n))]$ is an ordered sample of a $[0,1]-$-rectangular distribution, and we have

$$
p\left\{H(z(k)) \leq q_{\sigma}\right\}=\int_{0}^{q_{\sigma}} k\left(\begin{array}{l}
n \\
k
\end{array}\right) x^{k-1}(1-x)^{n-k} d x
$$

and, with partial integration,

$$
P\left\{H(z(k)) \leq q_{\sigma}\right\}=\sum_{i=k}^{n}\left(\begin{array}{c}
n \\
i
\end{array}\right) q_{\sigma}^{i}\left(1-q_{\sigma}\right)^{n-i} .
$$

With the help of Eq. (19), we get

$$
P\left\{z_{(k)} \leq \sigma \leq z_{(m)}\right\}=\sum_{i=k}^{m-1}\left(\begin{array}{l}
n \\
i
\end{array}\right) q_{\sigma}^{i}\left(1-q_{\sigma}\right)^{n-i} .
$$

The problem now can be formulated in the following way: find $k, m \in N^{*}$ such that

$$
\sum_{i=k}^{m-1}\left(\begin{array}{l}
n \\
i
\end{array}\right) q_{\sigma}^{i}\left(1-q_{\sigma}\right)^{n-i}=1-\alpha
$$

${ }^{*} \mathrm{~N}:=$ set of integers. 
can be fulfilled. Often it is not possible to solve Eq. exactly. Then the following procedure is helpful:

$$
\begin{aligned}
& \text { (i) choose } m-k \text { minimal } \\
& \text { (ii) } \sum_{i=k}^{m-1}\left({ }_{i}^{n}\right) q_{\sigma}^{i}\left(1-q_{\sigma}\right)^{n-i} \approx 1-\alpha \text {. }
\end{aligned}
$$

V. COMPARISON OF $\hat{\sigma}_{N}$ WITH TWO OTHER ESTIMATION METHODS

In this section, we look at two other estimation methods for the standard deviation of a normal distribution. 4 The first one is the classical maximum-likelihood estimation:

$$
s_{N}:=\sqrt{\sum_{i=1}^{N}\left(x_{i}-\bar{x}\right)^{2} / N},
$$

where

$$
\bar{x}=\sum_{i-1}^{N} x_{i} / N
$$

$S_{N}$ is a biased estimate, but

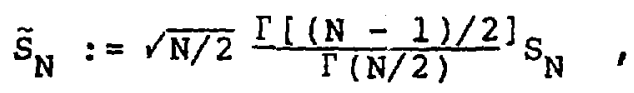

where $\Gamma(\cdot)$ denotes the gamma function, is an unbiased estimate. From Eq. (24) it follows that $s_{N}$ is asymptotically unbiased. Furthermore, we have ${ }^{5}$ 


$$
\operatorname{Var}\left(\tilde{S}_{N}\right)=\frac{\sigma^{2}}{2} \bar{N}+0\left(\frac{1}{N^{2}}\right),
$$

and as a result we see that $\tilde{S}_{N}$ is asymptotically efficient, because we have

$$
\frac{\operatorname{Var}\left(\tilde{S}_{N}\right)}{\sigma^{2} /(2 N)}=1+0\left(\frac{1}{N}\right) \text {. }
$$

From Eq. (26) it follows that $\mathrm{s}_{\mathrm{N}}$ is asymptotically efficient. second, we look at the mean deviation

$$
D_{N}:=\sqrt{\pi / 2} \sum_{i=1}^{N}\left|x_{i}-\bar{x}\right| / N
$$

In 1920, there was a disagreement between Eddington and Fisher about the relative merits of $S_{N}$ and $D_{N}$. We have

$$
E\left(\sqrt{\pi / 2} \sum_{i=1}^{N}\left|x_{i}-\mu\right| / N\right)=\sqrt{\pi / 2}\left[E\left(\left|x_{1}-\mu\right|\right)\right]=\sigma
$$

and

$$
\operatorname{var}\left(\sqrt{\pi / 2} \sum_{i=1}^{N}\left|x_{i}-\mu\right| / N\right)=(\pi-2) \sigma^{2} /(2 N),
$$

so that for $N$ large enough (that means $\overline{\mathrm{x}} \approx \mu$ ), ${ }^{D_{N}}$ is asymptotically unbiased with asymptotic variance $(\pi-2) \sigma^{2} /(2 \mathrm{~N})$.

In the following discussion, $\hat{\sigma}_{N}$ is compared with $s_{N}$ and ${ }^{D_{N}}$ under the given assumptions. As a criterion for the 
comparison, we choose the relative asymptotic efficiency. $\hat{\sigma}_{N}$ has the asymptotic variance

$$
\lim _{N \rightarrow \infty} N \cdot \operatorname{var}\left(\hat{\sigma}_{N}\right)=\frac{4 q_{\sigma}\left(1-q_{\sigma}\right)}{[h(\sigma)]^{2}} .
$$

Therefore, the asymptotic efficiency of $\sigma_{N}$ is

$$
\begin{aligned}
\lim _{N \rightarrow \infty} \operatorname{Eff}\left(\hat{\sigma}_{N}\right) & =\frac{\sigma^{2} /(2 N)}{2 q_{\sigma}\left(1-q_{\sigma}\right) /[h(\sigma)]^{2}} \\
& =2.59 \sigma^{2} .
\end{aligned}
$$

Because $S_{N}$ is asymptotically efficient, it follows that the relative asymptatic efficiency is

$$
\lim _{\mathrm{N} \rightarrow \infty} \operatorname{Eff}\left(\mathrm{S}_{\mathrm{N}}, \hat{\sigma}_{\mathrm{N}}\right)=\frac{\sigma^{2} / 2}{2.59 \sigma^{2}}=0.19 .
$$

For the relative asymptotic efficiency between $\hat{\sigma}_{N}$ and $D_{N}$, we have

$$
\lim _{N \rightarrow \infty} \operatorname{Eff}\left(D_{N}, \hat{\sigma}_{N}\right)=\frac{(\pi-2) \sigma^{2} / 2}{2.59 \sigma^{2}}=0.22 \text {. }
$$

Using the results of Eq. (29) and Eq. (30), one can find no argument to prefer $\hat{\sigma}_{N}$ if one takes the criterion of relative efficiency. The following considerations show that $\hat{\sigma}_{N}$ can be an attractive method nevertheless. 


\section{ROBUSTNESS}

In many practical examples, ${ }^{6,7}$ the theoretical assumption of a pure normal distribution does not hold. A reason for this can be radioactive contamination of the instruments during the measuring. Thus, bad measurements, called outliers, areipresent. among the data.

Now we will look at the three estimation methods $\hat{\sigma}_{N}, S_{N^{\prime}}$ and $D_{N}$ and check how they react to some changes in the data. For this consideration, we take as our first example some data from interlaboratory comparisons. ${ }^{6}$ one aim of these interlaboratory programs was to obtain some knowledge about the precision of measurement methods for ${ }^{238} \mathrm{U}$.

From these data we take the following first sample.

$$
\begin{aligned}
& x_{1}=2770, x_{2}=2811, x_{3}=2770, x_{4}=2732, x_{5}=2786, \\
& x_{6}=2770, x_{7}=2747, x_{8}=2779, x_{9}=2781, x_{10}=2757, \\
& x_{11}=2785, x_{12}=2747, x_{13}=2769, x_{14}=2755, x_{15}=2770, \\
& x_{16}=2766, x_{17}=2822, x_{18}=2764
\end{aligned}
$$

We can assume that the data are randomly ordered. Now ve construct a new sample of absolute differences:

$$
\begin{aligned}
& z_{1}=|2811-2770|=41, z_{2}=|2732-2770|=38 \\
& z_{3}=|2770-2786|=16, z_{4}=|2779-2747|=32 \\
& z_{5}=|2767-2781|=14, z_{6}=|2747-2785|=38 \\
& z_{7}=|2755-2769|=14, z_{8}=|2766-2770|=4 \\
& z_{9}=|2764-2822|=58 .
\end{aligned}
$$


With these data, we are able to construct the empirical distribution function $\mathrm{H}_{9}^{*}(t)$, shown in Fig. 2 .

For the estimate of $\sigma$, we get

$$
\hat{\sigma}_{18}=Q_{9}^{*}\left(q_{\sigma}\right)=z_{\left(\left[9 q_{\sigma}\right]+1\right)}=z_{(4+1)}=32 .
$$

A nice graphical interpretation of this estimation procedure is shown in Fig. 2 .

we get a 958 confidence interval for $\sigma$ with

$$
\sum_{i=2}^{7}\left(\begin{array}{l}
9 \\
i
\end{array}\right) 0.52^{i} 0.48^{9-i}=0.97 \text {. }
$$

It follows from this equation and from Eq. (2.2) that

$$
[2(2), 2(8)]=[14,41]
$$

is the wanted confidence interval.

In the following discussion, we use a so-called sensitivity curve $^{8}$ to show how the existence of one bad measurement has a different influence on the three estimation methods. We use our data set and let the value $x_{1}$ run from 2000 to 3400 . The estimates are illustrated in Fig. 3 , which shows very clearly that the cases $x_{1} \leq 2779$ or $x_{1}>2843$ have no further influence on the estimate for $\sigma$ using $\hat{\sigma}_{N}$, whereas in the other two cases the influence is still increasing.

In Fig. 3, we see the influence of only one bad measurement; the next question is how many bad measurements may be among the data without making the estimate useless. The problem is illustrated in this second example. ${ }^{6}$ 


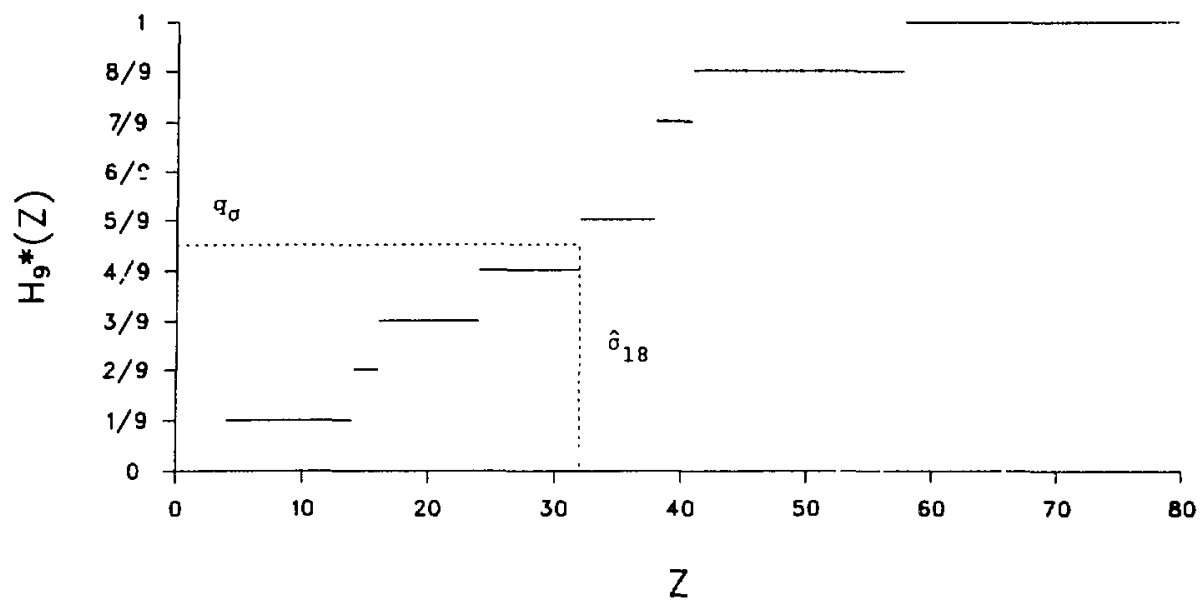

Fig. 2 .

Empirical distribution function $\mathrm{H}^{*}(t)$ and an estimate for $\sigma$ with $\hat{\sigma}_{18}$ using the data of the first example.

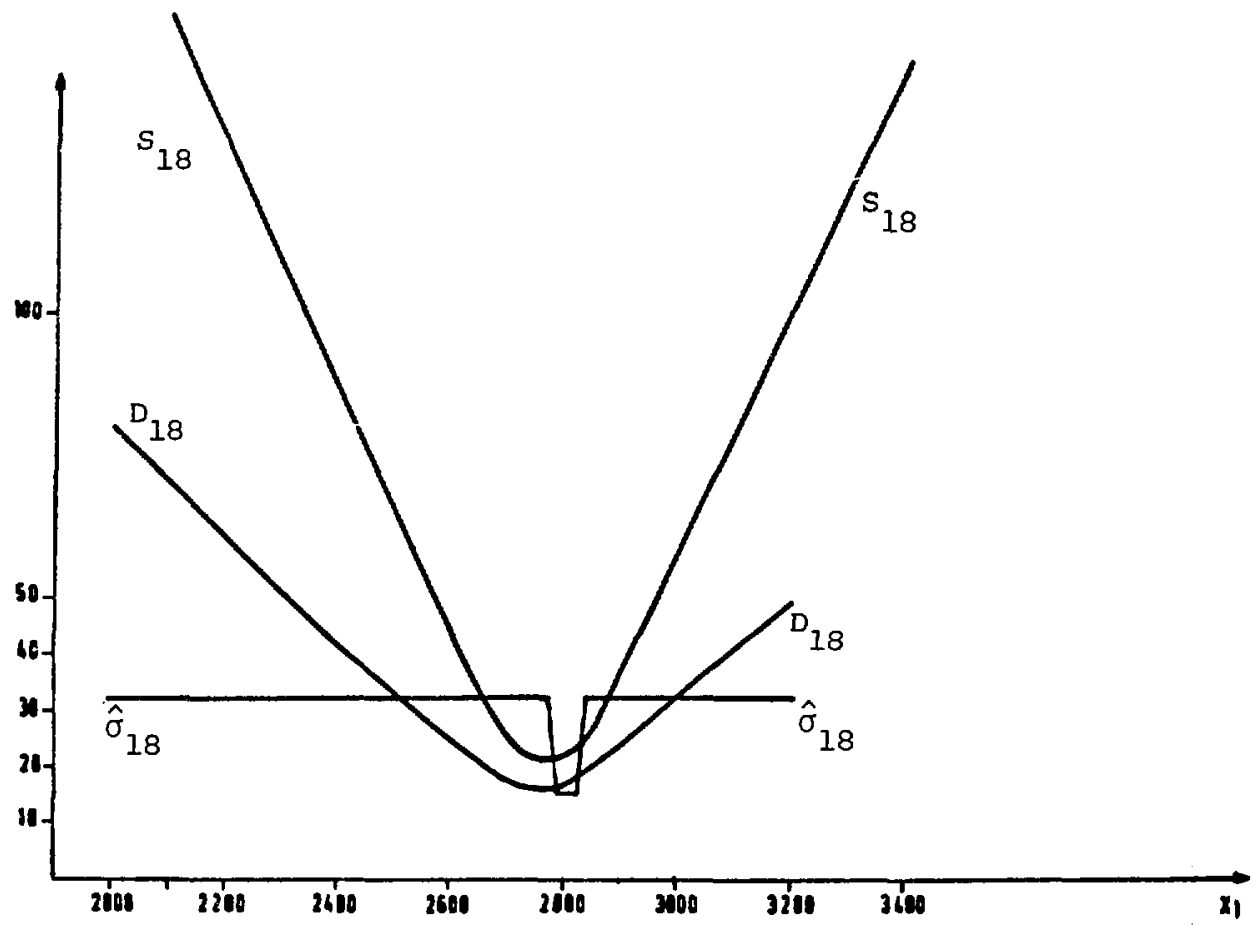

Fig. 3 .

Sensitivity curves for $\mathrm{S}_{18}, \mathrm{D}_{18}$, and $\hat{\sigma}_{18}$ at varying values of $x_{1}$ (data of first example). 


$$
\begin{aligned}
& x_{1}=(3530) \quad x_{2}=21.18 \quad x_{3}=2113 \quad x_{4}=2111 \\
& \mathrm{x}_{5}=2096 \quad \mathrm{x}_{6}=2119 \quad \mathrm{x}_{7}=2099 \quad \mathrm{x}_{8}=2103 \\
& x_{9}=2104 \quad x_{10}=2092 \quad x_{11}=(2307) \quad x_{12}=2107 \\
& x_{13}=(2382) \quad x_{14}=2129 \quad x_{15}=2094 \quad x_{16}=2081
\end{aligned}
$$

With these data, we will estimate the standard deviation using the three estimation methods (Fig. 4). We get

$$
\begin{aligned}
& S_{16}=358.09 \\
& D_{16}=242.33 \\
& \hat{\sigma}_{16}=23 .
\end{aligned}
$$

For $\hat{\sigma}_{16}$, we used

$$
\begin{aligned}
& z_{1}=|2118-3530|=1412 \\
& z_{2}=|2111-2113|=2 \\
& z_{3}=|2119-2096|=23 \\
& z_{4}=|2103-2099|=4 \\
& z_{5}=|2092-2104|=12 \\
& z_{6}=|2107-2307|=200 \\
& z_{7}=|2129-2382|=253 \\
& z_{8}=|2081-2094|=13 .
\end{aligned}
$$

The three results in Eq. (31) are very different. The question is why. We can get more insight into the problem if we estimate the standard deviation without the data in parentheses, that is, $x_{1}, x_{11}$, and $x_{13}$. Now we get

$$
\begin{aligned}
& S_{13}=13.02 \\
& D_{13}=12.83 \\
& \hat{\sigma}_{13}=15 .
\end{aligned}
$$




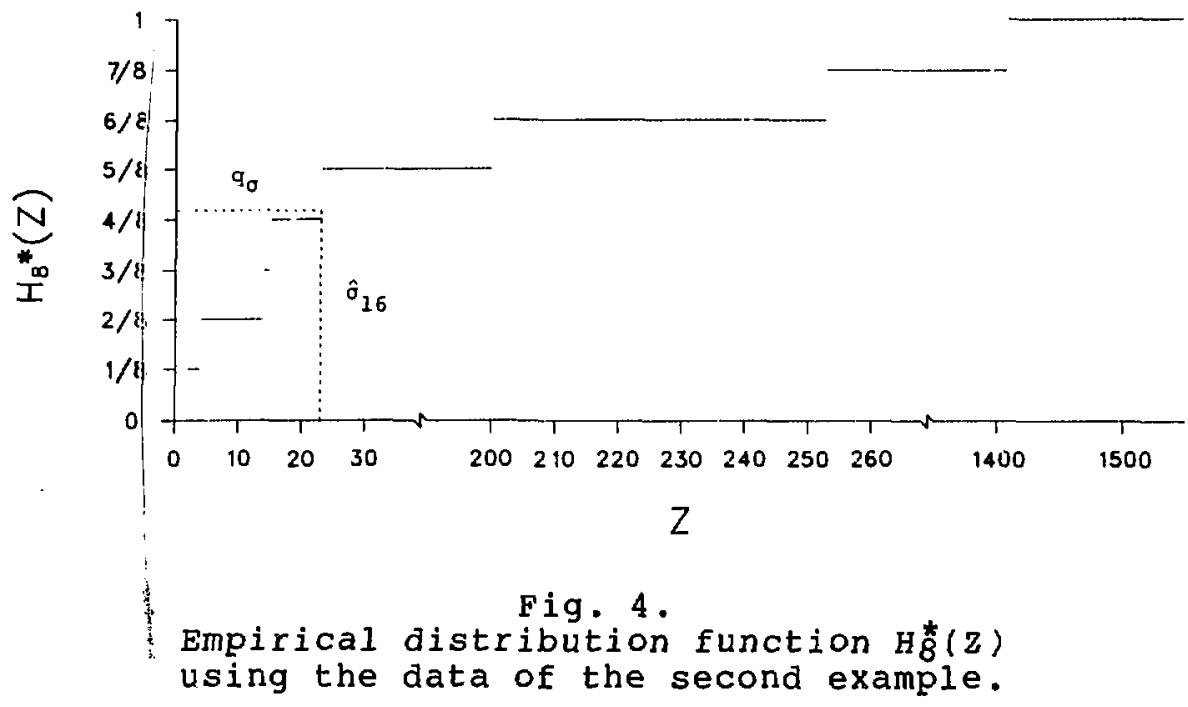

For $\hat{\sigma}_{13}$, we used

$$
\begin{aligned}
& z^{\prime}{ }_{1}=|2118-2113|=5 \\
& z^{\prime}{ }_{2}=|2111-2096|=15 \\
& z^{\prime}{ }_{3}=|2119-2099|=20 \\
& z^{\prime}{ }_{4}=|2104-2103|=1 \\
& z^{\prime}{ }_{5}=|2107-2092|=15 \\
& z_{6}{ }_{6}=|2129-2094|=35,
\end{aligned}
$$

and we have $\hat{\sigma}_{13}=z^{\prime}(4)$.

Looking now at Eq. (32), we see that in comparison with the data of Eq. (31) the results for $s_{N}$ and $D_{N}$ differ very much, whereas the results for $\hat{\sigma}_{N}$ differ very little:

We will now study some quantitative considerations that will illustrate the robustness of $\hat{\sigma}_{N}$. As an outlier model, we take the contaminated normal distribution; that is, we have a simple random sample $Y_{1}, Y_{2}, \ldots, Y_{N}$, where $Y_{1}$ has the density function

$$
g(y)=\alpha g_{0}(y)+(1-\alpha) g_{1}(y)
$$


with

$$
\begin{aligned}
& g_{0}(y)=\frac{1}{\sqrt{2 \pi \sigma}} \exp \left[\frac{(y-\mu)^{2}}{2 \sigma^{2}}\right], \\
& g_{1}(y)=\frac{1}{\sqrt{2 \pi k} \sigma} \exp \left[\frac{(y-\mu)^{2}}{2 k \sigma^{2}}\right] .
\end{aligned}
$$

With the help of Eq. (33), we get for the df $R(z)$ of $\left|Y_{1}-Y_{2}\right|$

$$
R(z)=\left\{\begin{array}{l}
\alpha^{2} \Phi\left(\frac{z}{\sqrt{2 \sigma}}\right)+4 \alpha(1-\alpha) \Phi\left(\frac{2}{\sqrt{k+1 \sigma}}\right)+2(1-\alpha)^{2} \Phi\left(\frac{z}{\sqrt{2 k \sigma}}\right)-1 \text { for } z \geq 0 . \\
0 \quad \text { otherwise. }
\end{array}\right.
$$

Using Eg. (34), we get

$$
\begin{aligned}
R(\sigma)= & \alpha^{2} \Phi(1 / \sqrt{2})+4 \alpha(1-\alpha) \Phi(1 / \sqrt{\mathrm{k}+1}) \\
& +2(1-\alpha)^{2} \Phi(1 / \sqrt{2 k})-1 .
\end{aligned}
$$

Now, if $k$ is sufficiently large, that is,

$$
\Phi(1 / \sqrt{\mathrm{k}+1}) \approx \Phi(1 / \sqrt{2 \mathrm{k}}) \approx 1 / 2,
$$

we have

$$
R(\sigma)=\alpha^{2}[2 \Phi(1 / \sqrt{2})-1]=\alpha^{2} q_{\sigma},
$$


and for $\alpha \approx 1$, we are able to write for sufficiently large $\mathrm{N}$

$$
\operatorname{var}\left(\hat{\sigma}_{N}\right)=\frac{q_{\sigma}\left(1-q_{\sigma}\right)}{N / 2} \frac{1}{R^{\prime}(\sigma)^{2}}=\frac{q_{\sigma}\left(1-q_{\sigma}\right) \pi \sigma^{2}}{N[A(k, \alpha)]^{2}},
$$

where

$$
\begin{aligned}
A \cdot(k, \alpha)= & \alpha^{2} \exp (-1 / 4) / \sqrt{2}+2 \alpha(1-\alpha) \exp \{-1 /[2(k+1)]\} / \sqrt{k+1} \\
& +(1-\alpha)^{2} \exp [-1 /(4 k)] / \sqrt{2 k} .
\end{aligned}
$$

Using Eq. (37), we see that

$$
\lim _{k \rightarrow \infty} \operatorname{var}\left(\hat{\sigma}_{N}\right)=\frac{q_{\sigma}\left(1-q_{\sigma}\right) \pi \exp (1 / 2)}{\alpha^{4}(N / 2)} \sigma^{2} .
$$

Equation (38) shows that the variance of $\hat{\sigma}_{\mathrm{N}}$ is limited also if the variance of the contamination goes to infinity.

Now we look at $s_{N}$ under the given assumptions. For $S_{N}$ ' we have

$$
s_{N}^{2}=\sum_{i=1}^{N}\left(Y_{i}-\bar{Y}\right)^{2} / N=\sum_{i, j=1}^{N}\left(Y_{i}-Y_{j}\right)^{2} /\left(2 N^{2}\right),
$$

and with the help of Eq. (39), we get

$$
E\left(S_{N}^{2}\right)=\frac{N(N-1)}{N^{2}}[\alpha+k(1-\alpha)] \sigma^{2} .
$$


Now, if we take $S_{N}$ for estimation of the standard deviation of $y_{1}$, we get $^{5}$

$$
\operatorname{Var}\left(S_{N}\right)=\frac{1}{4 N}\left\{3 \alpha-\alpha^{2}+k(1-\alpha)[2 k+(k-2) \alpha]\right\} \sigma^{2} .
$$

From Eq. (41), it follows at once that

$$
\operatorname{Var}\left(S_{N}\right)+\infty
$$

for $k+\infty$; that is, the variance of $S_{N}$ is not limited. If we now take $N$ sufficiently large, we get tha asymptotic relative efficiency between $s_{\mathrm{N}}$ and $\hat{\sigma}_{\mathrm{N}}$ :

$\lim _{N \rightarrow \infty} \operatorname{Eff}\left(S_{N}, \hat{\sigma}_{H}\right)=\frac{\left[3 \alpha-\alpha^{2}+k(1-\alpha)\right][2 k+(k-2) \alpha][A(k, \alpha)]}{4 \pi q_{\sigma}\left(1-q_{\sigma}\right)}(4 \dot{2})$

In the following discussion, we look at $D_{N}$ under the changed assumptions. The df $P(x)$ of $\left|Y_{1}-\mu\right|$ is, with Eq. (33),

$$
P(x)=\left\{\begin{array}{l}
2 \alpha \Phi(x / \sigma)+2 \alpha(1-\alpha) \Phi(x / \sqrt{k} \sigma)-1 \text { for } x \geq 0, \\
0 \quad \text { otherwise. }
\end{array}\right.
$$

With Eq. (43), we get

$$
E\left(\left|Y_{1}-\mu\right|\right)=[\alpha+\sqrt{k}(1-\alpha)] \sqrt{2 / \pi} \sigma,
$$


and

$$
\operatorname{Var}\left(\left|Y_{i}-\mu\right|\right)=\frac{\pi}{2} \bar{N}\left[\alpha+(1-\alpha) k-\frac{2}{\pi}\right] \sigma^{2},
$$

or

$$
\operatorname{Var}\left(D_{N}\right)=\frac{\pi}{2} \bar{N}\left[\alpha+(1-\alpha) k-\frac{2}{\pi}\right] \sigma^{2} \text {. }
$$

A direct consequence of Eq. (45) is

$$
\operatorname{Var}\left(D_{N}\right) \rightarrow \infty
$$

for $k \rightarrow \infty$; that is, the variance of $D_{N}$ is also not limited. For the relative asymptotic efficiency between $D_{N}$ and $\hat{\sigma}_{N}$ we get

$$
\lim _{N \rightarrow \infty} \operatorname{Eff}\left(D_{N}, \hat{\sigma}_{N}\right)=\frac{[A(k, \alpha)]^{2}[\alpha+(1-\alpha) k-2 / \pi]}{2 q_{\sigma}\left(1-q_{\sigma}\right)} .
$$

Table I gives an overview of the asymptotic relative efficiencies between $\hat{\sigma}_{\mathrm{N}}, \mathrm{S}_{\mathrm{N}}$, and $D_{\mathrm{N}}$ for some values of $\alpha$ and $\mathrm{k}$.

VII. CONCLUDING REMARKS

The considerations and examples in this report show that $\hat{\sigma}_{\mathrm{N}}$ is a robust estimation method for the standard deviation of a normal distribution. Now we have to answer the question: How do we use this method with its nice graphical interpretation? Because $\hat{\sigma}_{N}$ has a low efficiency under a pure normal distribution, one should not use it exclusively. But its robustness in 
TABLE I

ASYMPTOTIC RELATIVE EFFICIENCIES BETWEEN $\sigma_{N}, s_{N}$, and $D_{N}$

\begin{tabular}{|c|c|c|c|c|}
\hline & & $\alpha$ & $E I^{a}$ & $E 2^{b}$ \\
\hline \multirow[t]{7}{*}{$\mathbf{k}$} & $=4$ & 0.99 & 0.23 & 0.24 \\
\hline & & 0.98 & 0.26 & 0.25 \\
\hline & & 0.96 & 0.33 & 0.28 \\
\hline & & 0.95 & 0.36 & 0.30 \\
\hline & & 0.90 & 0.51 & 0.36 \\
\hline & & 0.80 & 0.74 & 0.47 \\
\hline & & 0.75 & 0.83 & 0.52 \\
\hline \multirow[t]{7}{*}{$k$} & $=9$ & 0.99 & 0.40 & 0.26 \\
\hline & & 0.98 & 0.60 & $0 \cdot 31$ \\
\hline & & 0.96 & 0.98 & 0.39 \\
\hline & & 0.95 & 1.15 & 0.42 \\
\hline & & 0.90 & 1.91 & 0.59 \\
\hline & & 0.80 & 2.96 & 0.82 \\
\hline & & 0.75 & 3.29 & 0.90 \\
\hline \multirow[t]{7}{*}{$\mathbf{k}$} & $=16$ & J.99 & 0.88 & 0.30 \\
\hline & & 0.98 & 1.53 & 0.38 \\
\hline & & 0.96 & 2.73 & 0.53 \\
\hline & & 0.95 & 3.29 & 0.60 \\
\hline & & 0.90 & 5.61 & 0.90 \\
\hline & & 0.80 & 8.44 & 1.27 \\
\hline & & 0.75 & 9.16 & $1 \cdot 38$ \\
\hline \multirow[t]{7}{*}{$\mathbf{k}$} & $=25$ & 0.99 & 1.90 & 0.36 \\
\hline & & 0.98 & 3.51 & 0.49 \\
\hline & & 0.96 & 6.45 & 0.72 \\
\hline & & 0.95 & 7.78 & 0.83 \\
\hline & & 0.90 & 13.24 & 1.29 \\
\hline & & 0.80 & 19.30 & 1.82 \\
\hline & & 0.75 & 20.53 & 1.95 \\
\hline \multirow[t]{7}{*}{$\mathbf{k}$} & $=36$ & 0.99 & 3.76 & 0.42 \\
\hline & & 0.98 & 7.11 & 0.61 \\
\hline & & 0.96 & 13.17 & 0.96 \\
\hline & & 0.95 & 15.90 & 1.11 \\
\hline & & 0.90 & 26.87 & 1.76 \\
\hline & & 0.80 & 38.19 & 2.47 \\
\hline & & 0.75 & 40.03 & 2.61 \\
\hline
\end{tabular}

$\overline{a \operatorname{El}=\lim _{N \rightarrow \infty} \operatorname{Eff}}\left(S_{N}, \hat{\sigma}_{N}\right)$.

b E2 $=\lim _{N \rightarrow \infty} \operatorname{Eff}\left(D_{N}, \hat{\sigma}_{N}\right)$. the presence of outliers suggests the following uses, which Hampel ${ }^{9}$ also proposed:

(1) crude estimation for the standard deviation, and

(2) a control for more exact calculations.

Another advantage of $\hat{\sigma}_{N}$ is that one has avoided using outlier criteria with the possibility of wrong decisions.

\section{ACKNOWLEDGMENTS}

I want to thank J. P. Shipley and $A$. S. Goldman in the Safeguards systems Group at Los Alamos National Laboratory for their comments and help to improve this report.

\section{REFERENCES}

1. E. Parzen, "A Density-Quantile Function Perspective on Robust Estimation," in Robustness in statistics, R. C. Launer and G. N. Wilkinson, Eds. (Academic Press, New York, 1979), pp. $237-256$.

2. M. Fisz, Wahrscheinlichkeitsrechnung und Mathematische statistik (VEB Deutscher Verlag der Wissenschaften, Berlin, 1970). 
3. H. Buening and G. Trenkler, Nichtparanetrische Statistische Methoden (Walter de Gruyter, Berlin, New York, 1978).

4. P. Huber, Robust Statistical procedures (Society for Industrial and Applied Mathematics, Philadelphia, 1977), Regional Conference Series in Applied Mathematics, 27, 1977.

5. V. Rohatgi, Ar Introduction to Probability Theory and Mathematical statistics (John Wiley \& Sons, New York, 1976).

6. W. Beyrich and E. Drosselmeyer, "The Interlaboratory Experiment IDA-72 on Mass Spectrometric Isotope Dilution Analysis," KfK 1905, EUR-5203e (1975).

7. W. Beyrich and G. Spannagel, "The AS-75 Interlaboratory Experiment on the Alpha spectrometric Determination of Pu-238," Part I, Kfk 2860, EUR 6400e (1979).

8. J. W. Tukey, Exploratury Data Analysis, Preliminary Ed. (Addison-Wesley, Reading, Massachusetts, 1970).

9. F. Hampel, "The Influence Curve and Its Role in Robust Estimation," J. Am. Stat. Assoc. $69(346), 383-393$ (1974). 